磺酰肼和二硫缩烯酮区域选择性环化反应合成 3-烷硫基取代吡唑

\author{
李 毅 万结平* \\ (江西师范大学化学化工学院 南昌 330022)
}

\begin{abstract}
摘要 以磺酰肼和二硫缩烯酮为起始物, 在廉价易得的硫酸氢钠作为催化剂条件下, 建立了二者之间区域选择性环化 合成 3-烷硫基取代的吡唑类化合物的方法. 在 1,4-二氧六环作为介质和 $80{ }^{\circ} \mathrm{C}$ 加热条件下进行，高效合成了一系列 $N$ 磺酰基结构的 3 -烷硫基吡唑化合物.
\end{abstract}

关键词 二硫缩烯酮; 磺酰肼; 环化; 区域选择性; 3-烷硫基吡夾

\title{
Synthesis of 3-Alkylthiol Pyrazoles via Regioselective Annulation Reactions of Sulfonyl Hydrazines and Ketene Dithioacetals
}

\author{
Li, Yi Wan, Jieping* \\ (College of Chemistry and Chemical Engineering, Jiangxi Normal University, Nanchang 330022)
}

\begin{abstract}
By using sulfonyl hydrazines and ketene dithioacetals as starting materials, the regioselective annulation providing 3 -alkylthiolated pyrazoles has been realized in the low cost and easily available $\mathrm{NaHSO}_{4}$ catalytst. The reactions were realized in 1,4-dioxane medium and $80{ }^{\circ} \mathrm{C}$ heating, whereby a seris of 3-alkylthiol pyrazoles possessing $N$-sulfonyl structure have been efficiently synthesized.

Keywords ketene dithioacetal; sulfonyl hydrazine; annulation; regioselectivity; 3-alkylthiopyrazole
\end{abstract}

吡唑是最常见的芳杂环结构之一，在化学、材料、 医药等多个制造和研究领域具有广泛且重要的应用. 例 如，吡唑结构作为高效导向基团可以辅助过渡金属催化 实现多种碳-氢键活化反应 ${ }^{[1]}$, 还可以通过结构中的螯 合位点和金属离子形成结构多样、潜在功能丰富的有机 金属复合物 ${ }^{[2]}$. 此外，吡唑环在功能材料分子中也常作 为关键结构存在 ${ }^{[3]}$. 更重要的是, 许多以吡唑为主体结 构的有机小分子具有丰富多样的生物药物活性, 对于药 物的研发和功效改进均具有重要意义. 例如, 许多吡唑 的衍生物在抗癌、抗菌、抗美洲雉虫病、抗炎、除草以 及杀虫等方面展示了高度多样的生物药物活性 ${ }^{[4]}$. 因此, 对于吡唑类化合物, 尤其是结构多样性的吡唑类化合物 的合成也成为有机合成化学研究的重要课题 ${ }^{[5]}$.

对于常规的吡唑类化合物, 可通过一些经典方法如 肼和 1,3-二酮类类化合物的缩合环化实现 ${ }^{[6]}$. 在此基础 上, 多种不同的合成方法如烯胺酮和肼环化串联反
应 ${ }^{[7]}$ 、基于 $\mathrm{N}-\mathrm{N}$ 键构建的多组分反应 ${ }^{[8]}$ 、贫电子烯/炔 衍生物参与的吡唑环化反应 ${ }^{[9]}$ 、重氮作为双氮杂片段供 体的环化反应 ${ }^{[10]}$ 、活泼亚甲基-醛缩合以及肼环化串联 反应 ${ }^{[11]}$ 、过渡金属催化的交叉偶联串联反应 ${ }^{[12]}$ 和醛腙/

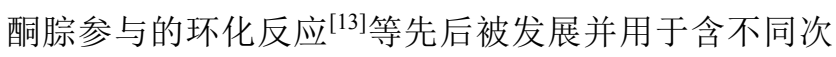
级结构的吡唑类化合物合成. 然而, 由于吡唑类化合物 重要的已知和潜在应用前景, 发展可用于合成具有新型 官能结构的吡唑类化合物的方法仍然是有机合成研究 的重点课题之一. 例如, 含有杂原子结构侧链取代的杂 环化合物因其在发现活性先导化合物及其它功能分子 方面的优势, 近期成为有机合成研究的热点课题 ${ }^{[14-15]}$. 基于我们小组前期在杂侧链杂环化合物合成方面的结

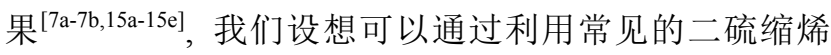
酮作为底物 ${ }^{[16]}$, 通过其中一个烯基碳一硫键断裂环化, 同时另一硫官能团保留的反应模式直接实现含硫侧链 的吡唑合成. 值得一提的是, Ila 和 Junjappa 等 ${ }^{[17]}$ 在早期

\footnotetext{
* Corresponding author. E-mail: wanjieping@jxnu.edu.cn

Received May 10, 2020; revised June 16, 2020; published online July 15, 2020.

Dedicated to the 40th anniversary of Chinese Journal of Organic Chemistry

Project supported by the National Natural Science Foundation of China (No. 21861019).

国家自然科学基金(No. 21861019)资助项目.
} 
曾研究过二硫缩烯酮和芳基肼在等物质的量叔丁醇钾 促进下的反应，通过在叔丁醇中回流可得到 5-甲硫基吡 唑(Scheme 1, A). 而本研究工作, 通过篮选的催化条件, 以催化量的 $\mathrm{KHSO}_{4}$ 在二氧六环介质中, 加热二硫缩烯 酮和磺酰肼, 以不同的区域选择性实现了 3-烷硫基吡唑 类化合物的合成(Scheme 1, B).

(A) Previous

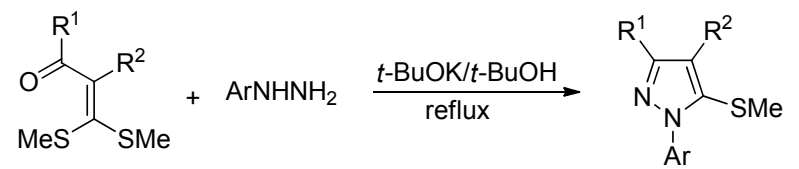

(B) This work<smiles>[R]C(=O)C([R18])=C([R15])[R15]</smiles>

图式 1 5-甲硫基和 3-烷硫基代吡唑类化合物的合成 Scheme 1 Synthesis of 5-methylthio- and 3-alkylthio-pyrazoles

\section{1 结果与讨论}

研究开始, 选择了二硫缩烯酮 1a 和对甲基苯磺酰 肼 (2a)作为起始物, 进行反应条件的篎选研究, 结果如 表 1 所示. 首先, 采用 1,4-二氧六环作为介质, 反应温度 为 $65{ }^{\circ} \mathrm{C}$ 时对不同的酸如 $\mathrm{KHSO}_{4}$ 、草酸、氯化铁、氯化 铜、溴化亚酮、三氟甲磺酸进行了篎选. 等物质的量的 酸条件下的反应证实 $\mathrm{KHSO}_{4}$ 对生成产物 $\mathbf{3 a}$ 的反应具有 最好的效果(Entries $1 \sim 6$, 表 1). 随后, 采用一系列具有 不同极性的常见有机溶剂作为介质, 结果显示二氧六环 是该反应的最优介质(Entries $7 \sim 11$, 表 1). 接下来, 也 考察了 $\mathrm{KHSO}_{4}$ 负载量对反应的影响, 结果显示仅需要 0.2 equiv. 的 $\mathrm{KHSO}_{4}$ 作为催化剂就可以获得和等物质的 量添加时近似的结果(Entries 12 14, 表 1). 接下来, 对 原料比例进行调整时, 发现以 $\mathbf{1 a}$ 稍过量, 即在 1.2 倍物 质的量时, 可获得和 1.5 倍物质的量 $1 \mathrm{a}$ 以及等物质的量 $\mathrm{KHSO}_{4}$ 反应等同的产率(Entry 15 , 表 1 ). 因此, 以此为 基础, 对反应温度进行了优化, 在反应温度升高至 $80{ }^{\circ} \mathrm{C}$ 时以优秀的产率获得了目标产物(Entries 16, 17, 表 1).

在优化反应条件的基础上，对反应的适用范围进行 了考察. 如表 2 所示, 采用不同的二硫缩烯酮类底物 $\mathbf{1}$ 以及磺酰肼 2 在优化条件下进行反应, 反应结果总体较 好. 首先, 芳基结构上含有烷基、烷氧基、卤素等常见 官能团以及无取代的苯基官能化二硫缩烯酮和对甲苯 磺酰肼反应均可以良好到优秀的产率得到目标产物 $(3 \mathbf{a} \sim 3 \mathbf{h}$, 表 2). 此外, 䒺基结构(3i，表 2)以及双取代苯
表 1 反应条件优化 ${ }^{a}$

Table 1 Optimization of reaction conditions

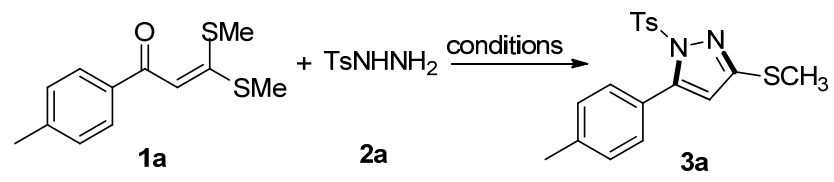

\begin{tabular}{ccccc}
\hline Entry & Acid & Solvent & $T /{ }^{\circ} \mathrm{C}$ & Yield $^{b} \%$ \\
\hline 1 & $\mathrm{KHSO}_{4}$ & Dioxane & 65 & 78 \\
2 & $(\mathrm{COOH})_{2}$ & Dioxane & 65 & 74 \\
3 & $\mathrm{FeCl}_{3}$ & Dioxane & 65 & 36 \\
$4^{c}$ & $\mathrm{CuCl}_{2}$ & Dioxane & 65 & 30 \\
5 & $\mathrm{CuBr}$ & Dioxane & 65 & 48 \\
6 & $\mathrm{TfOH}$ & Dioxane & 65 & Trace \\
7 & $\mathrm{KHSO}_{4}$ & DMF & 65 & $\mathrm{nr}$ \\
8 & $\mathrm{KHSO}_{4}$ & DMSO & 65 & $\mathrm{nr}$ \\
9 & $\mathrm{KHSO}_{4}$ & MeCN & 65 & 75 \\
10 & $\mathrm{KHSO}_{4}$ & PhMe & 65 & 44 \\
11 & $\mathrm{KHSO}_{4}$ & EtOH & 65 & $\mathrm{Trace}$ \\
$12^{d}$ & $\mathrm{KHSO}_{4}$ & Dioxane & 65 & 76 \\
$13^{e}$ & $\mathrm{KHSO}_{4}$ & Dioxane & 65 & 74 \\
$14^{f}$ & $\mathrm{KHSO}_{4}$ & Dioxane & 65 & 74 \\
$15^{f, g}$ & $\mathrm{KHSO}_{4}$ & Dioxane & 65 & 78 \\
$16^{f, g}$ & $\mathrm{KHSO}_{4}$ & Dioxane & 50 & 72 \\
$17^{f, g}$ & $\mathrm{KHSO}_{4}$ & Dioxane & 80 & 86 \\
\hline
\end{tabular}

${ }^{a}$ General conditions: 1a $(0.3 \mathrm{mmol}), \mathbf{2 a}(0.2 \mathrm{mmol})$, acid $(0.2 \mathrm{mmol})$, in solvent $(2 \mathrm{~mL})$, stirred for $2 \mathrm{~h} .{ }^{b}$ Isolated yield. ${ }^{c} \mathrm{CuCl}_{2} \cdot 2 \mathrm{H}_{2} \mathrm{O}$ was used. ${ }^{d}$ With 0.4 mmol of acid. ${ }^{e}$ With $0.1 \mathrm{mmol}$ of acid. ${ }^{f}$ With 0.04 mmol of acid. ${ }^{g} \mathbf{1 a}(0.2$ $\mathrm{mmol})$ and $\mathbf{2 a}(0.24 \mathrm{mmol})$.

表 $2 N$-磺酰基-3-烷硫基吡唑类化合物的合成反应 ${ }^{a, b}$

Table 2 Synthesis of $N$-sulfonyl-3-alkylthiopyrazoles

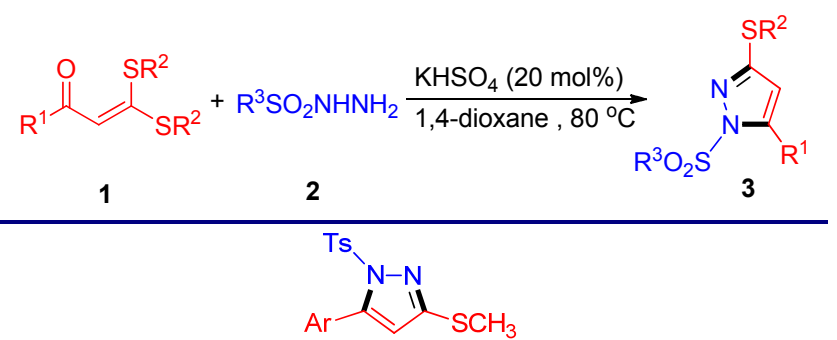

3a, $\mathrm{Ar}=p$-toyl, $86 \%$ 3b, $\mathrm{Ar}=\mathrm{Ph}, 83 \%$

3f, $\mathrm{Ar}=4-\mathrm{BrC}_{6} \mathrm{H}_{4}, 80 \%$ $3 \mathrm{~g}, \mathrm{Ar}=m$-toyl, $89 \%$

3c, $\mathrm{Ar}=4-\mathrm{MeOC}_{6} \mathrm{H}_{4}, 90 \%$ 3h, $\mathrm{Ar}=3-\mathrm{ClC}_{6} \mathrm{H}_{4}, 77 \%$ 3d, $\mathrm{Ar}=4-\mathrm{FC}_{6} \mathrm{H}_{4}, 85 \% \quad 3 \mathbf{3 i}, \mathrm{Ar}=2$-naphthyl, $74 \%$

3e, $\mathrm{Ar}=4-\mathrm{ClC}_{6} \mathrm{H}_{4}, 81 \%$

$3 \mathbf{j}, \mathrm{Ar}=3,4-\mathrm{Cl}_{2} \mathrm{C}_{6} \mathrm{H}_{3}, 72 \%$
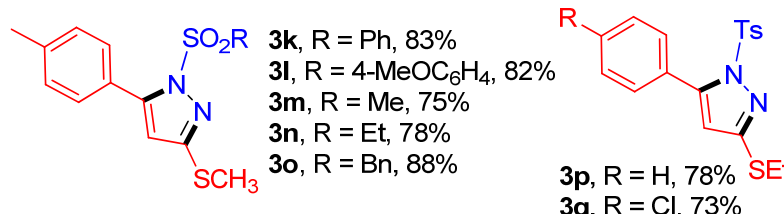

$3 p, \mathrm{R}=\mathrm{H}, 78 \%$ SEt $3 q, \mathrm{R}=\mathrm{Cl}, 73 \%$

${ }^{a}$ Reaction conditions: $1(0.2 \mathrm{mmol}), 2(0.24 \mathrm{mmol}), \mathrm{KHSO}_{4}(0.04 \mathrm{mmol})$ in 1,4-dioxane $(2 \mathrm{~mL})$, stirred at $80{ }^{\circ} \mathrm{C}$ for $2 \mathrm{~h} .{ }^{b}$ Isolated yield.

基(3j，表 2)结构的缩烯酮类化合物也对合成反应表现 了良好的兼容性. 另一方面，不同的磺酰肼底物，包括 芳基磺酰肼 $(3 \mathbf{k} \sim 31$ ，表 2)以及烷基磺酰肼 $(3 \mathrm{~m}, \mathbf{3 0}$ ，表 2) 均顺利用于该反应合成, 到了相应的吡唑产物. 更值得 
一提的是，除了甲硫基结构的二硫缩烯酮，乙硫基结构 的二硫缩烯酮也可以参与反应得到相应的产物(3p, 3q, 表 2). 这些结果显示了该合成方法对不同类型底物的高 度兼容性. 所有产物产率大体为良好到优秀, 目前结果 中取代基性质未表现出对反应结果的显著影响. 而当采 用烷基酮衍生的二硫缩烯酮 $\left(\mathrm{R}^{1}=\mathrm{R}^{2}=\mathrm{Me}\right)$ 和 $2 \mathrm{a}$ 反应时, 未得到相应的吡唑产物.

有趣的是, 当使用乙酰丙酮衍生的二硫缩烯酮 4 作 为底物和对甲苯磺酰肼反应时, 在标准条件下以优秀产 率得到全取代的吡唑产物 $3 \mathrm{r}$, 进一步证实了本方法在 合成结构多样性烷硫基吡唑类化合物方面的优势 (Eq. 1). 此外, 采用 1-苯基-1,3-丁二酩衍生的二硫缩烯酮作 为底物进行反应时, 因其结构中存在两个不同但均具有 反应活性的酮羰基, 反应得到的较为复杂的混合物. 作 为新型区域选择性合成方法, 本研究所合成的所有吡唑 产物均为新化合物, 对代表性化合物 3a 的单晶衍射分 析进一步确证了所合成产物的结构(图 1) ${ }^{[18]}$.
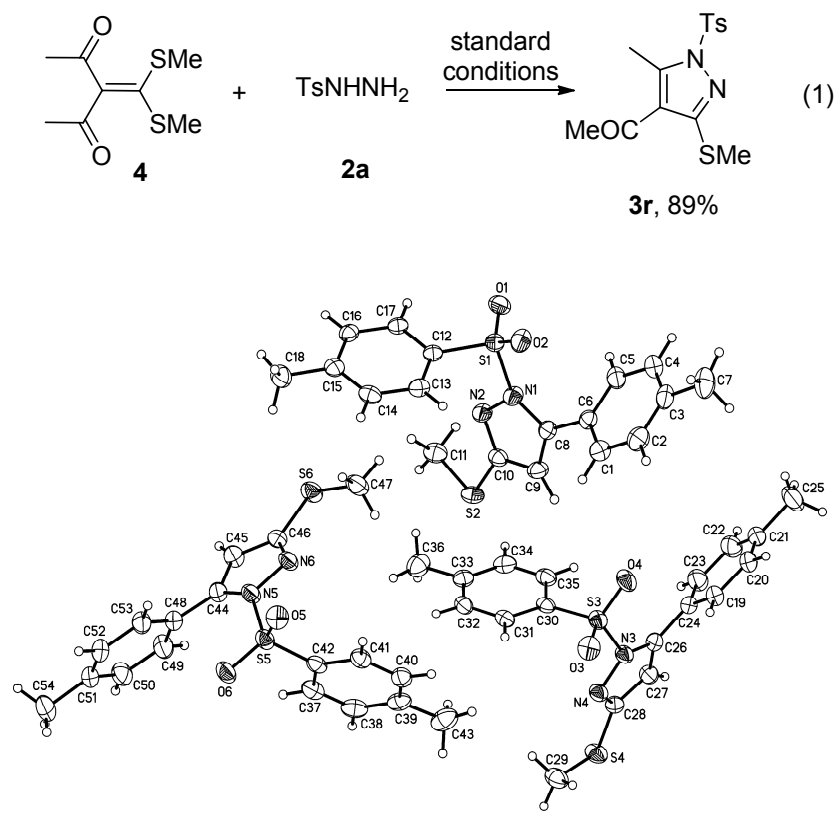

图 1 产物 3a 的单晶结构(三分子共聚结构)

Figure 1 Single crystal structure of product 3a (three commensal molecules)

根据实验结果, 我们对可能的反应机理进行了推 测. 如 Scheme 2 所示, 弱酸性的硫酸氢钾质子选择性和 亲核性较强的硫位点作用, 将二硫缩烯酮活化为结构 $\mathbf{A}$, 经历异构化得到的结构 $\mathbf{B}$ 被磺酰肼中的末端氮进攻 得到中间体 C. C 消除硫酚得到中间体 $\mathbf{D}$, 该中间体经历 分子内氨基对羰基的亲核加成得到吡唑啉中间体 $\mathbf{E}$ ，该 中间体在酸催化下脱水得到吡唑啉离子 F. F 再通过分 子内电子转移并释放出质子即得到目标产物 $\mathbf{3}$.

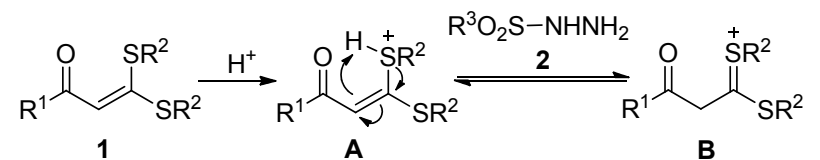

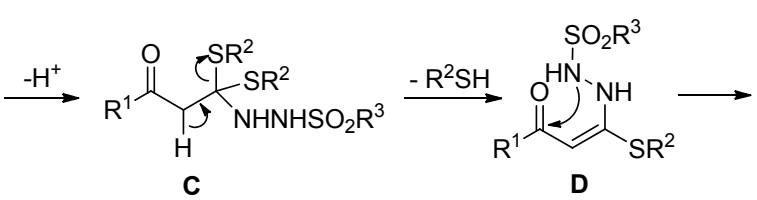<smiles>[R1]OS(=O)(=O)n1nc([R16])cc1[R1]</smiles>

图式 2 可能的反应机理

Scheme 2 Proposed reaction mechanism

\section{2 结论}

采用简单易得的二硫缩烯酮和磺酰肼为起始物，以 廉价的硫酸氢钠作为催化剂实现了二者之间的吡唑环 化反应, 得到了 3 -烷硫基化的吡唑衍生物, 结果展示了 和文献生成 5-甲硫基吡唑不一样的区域选择性. 此外, 该区域选择性的方法在底物适用性上表现出独特的优 势. 不同类型的烷基二硫缩烯酮和芳基/烷基磺酰肼均 可兼容于该催化方法，高效生成了结构多样性的烷硫基 吡唑产物. 研究结果为新型硫侧链官能化的吡唑类化合 物的合成提供了实用方法.

\section{3 实验部分}

\section{1 仪器与试剂}

本工作中合成吡唑的实验均在敞口条件下进行. 本 实验所使用二硫缩烯酮均为参考文献[18]以苯乙酮为原 料所合成. 此外, 除对甲苯磺酰肼与苯基磺酰肼外的其 它磺酰肼底物也参考文献[19]方法以磺酰氯与水合肼合 成. 其它化学试剂和溶剂均为商业购得, 使用前未经再 次处理. ${ }^{1} \mathrm{H}$ NMR 和 ${ }^{13} \mathrm{C}$ NMR 在 Bruker AVANCE 400 核 磁共振仪在 $\mathrm{CDCl}_{3}$ 溶剂中测试, 化学位移以 TMS 为内 标, ${ }^{1} \mathrm{H}$ NMR 和 ${ }^{13} \mathrm{C}$ NMR 测试频率分别为 400 和 100 MHz. 高分辨质谱结果在配置了 TOF 离子源的质谱仪 在 ESI 模式下测得. 熔点通过 X-4A 熔点仪测试获得, 测 试前未经额外校正.

\section{2 实验方法}

在 $25 \mathrm{~mL}$ 圆底烧瓶中加入二硫缩烯酮 1 (0.2 $\mathrm{mmol})$ 、 磺酰肼 $2(0.24 \mathrm{mmol})$ 以及硫酸氢钾 $(0.04 \mathrm{mmol})$, 最后加 入 $2 \mathrm{~mL}$ 的 1,4 -二氧六环. 所得混合物在 $80{ }^{\circ} \mathrm{C}$ 空气氛围 下回流反应 $2 \mathrm{~h}$. 待冷却至室温后, 往烧瓶中加入 $5 \mathrm{~mL}$ 的乙酸乙酯与 $5 \mathrm{~mL}$ 的水. 所得悬乳液以乙酸乙酯萃取 
$(8 \mathrm{~mL} \times 3)$. 萃取所得有机相合并后以无水硫酸钠干燥, 过滤. 滤液中的溶剂通过减压蒸馏除去. 残余物通过硅 胶柱色谱以石油醚和乙酸乙酯混合物 $(V: V=10: 1)$ 淋 洗提纯得到纯产物.

3-甲硫基-5-对甲基苯基-1-对甲苯磺酰基- $1 H$-吡唑 (3a): 白色固体, m.p. 91.2 92.7 ${ }^{\circ} \mathrm{C} ;{ }^{1} \mathrm{H}$ NMR (400 MHz, $\left.\mathrm{CDCl}_{3}\right) \delta: 7.56(\mathrm{~d}, J=8.4 \mathrm{~Hz}, 2 \mathrm{H}), 7.30(\mathrm{~d}, J=8.0 \mathrm{~Hz}$, 2H), $7.22(\mathrm{~d}, J=7.2 \mathrm{~Hz}, 4 \mathrm{H}), 6.12(\mathrm{~s}, 1 \mathrm{H}), 2.54(\mathrm{~s}, 3 \mathrm{H})$, $2.42(\mathrm{~s}, 3 \mathrm{H}), 2.39(\mathrm{~s}, 3 \mathrm{H}) ;{ }^{13} \mathrm{C} \mathrm{NMR}\left(100 \mathrm{MHz}, \mathrm{CDCl}_{3}\right) \delta$ : $154.5,150.3,145.2,139.7,134.6,129.7,129.5,128.5$, 127.9, 126.3, 111.1, 21.7, 21.5, 14.2; ESI-HRMS calcd for $\mathrm{C}_{18} \mathrm{H}_{19} \mathrm{~N}_{2} \mathrm{O}_{2} \mathrm{~S}_{2}[\mathrm{M}+\mathrm{H}]^{+}$359.0882, found 359.0882.

3-甲硫基-5-苯基-1-对甲苯磺酰基- $1 H$-吡唑(3b): 白 色固体, m.p. 105.3 106.7 ${ }^{\circ} \mathrm{C} ;{ }^{1} \mathrm{H}$ NMR (400 MHz, $\left.\mathrm{CDCl}_{3}\right) \delta: 7.56(\mathrm{~d}, J=8.4 \mathrm{~Hz}, 2 \mathrm{H}), 7.48 \sim 7.38(\mathrm{~m}, 5 \mathrm{H})$, $7.22(\mathrm{~d}, J=8.1 \mathrm{~Hz}, 2 \mathrm{H}), 6.16(\mathrm{~s}, 1 \mathrm{H}), 2.55(\mathrm{~s}, 3 \mathrm{H}), 2.40$ (s, $3 \mathrm{H}) ;{ }^{13} \mathrm{C}$ NMR (100 MHz, $\left.\mathrm{CDCl}_{3}\right) \delta: 150.0,145.2,129.8$, 129.6, 129.5, 129.2, 127.9, 127.8, 111.3, 21.7, 14.2; ESI-HRMS calcd for $\mathrm{C}_{17} \mathrm{H}_{17} \mathrm{~N}_{2} \mathrm{O}_{2} \mathrm{~S}_{2}[\mathrm{M}+\mathrm{H}]^{+} 345.0726$, found 345.0726 .

3-甲硫基-5-对甲氧基苯基-1-对甲苯磺酰基- $1 H$-吡 唑(3c): 白色固体, m.p. $179.5 \sim 180.6{ }^{\circ} \mathrm{C} ;{ }^{1} \mathrm{H}$ NMR (400 $\left.\mathrm{MHz}, \mathrm{CDCl}_{3}\right) \delta: 7.54(\mathrm{~d}, J=8.4 \mathrm{~Hz}, 2 \mathrm{H}), 7.33$ (d, $J=$ $8.8 \mathrm{~Hz}, 2 \mathrm{H}), 7.20$ (d, $J=8.0 \mathrm{~Hz}, 2 \mathrm{H}), 6.94$ (d, $J=8.8 \mathrm{~Hz}$, 2H), 6.10 (s, 1H), $3.86(\mathrm{~s}, 3 \mathrm{H}), 2.53(\mathrm{~s}, 3 \mathrm{H}), 2.38(\mathrm{~s}, 3 \mathrm{H})$; ${ }^{13} \mathrm{C}$ NMR (100 MHz, $\left.\mathrm{CDCl}_{3}\right) \delta: 160.7,154.5,150.2,145.1$, 134.6, 131.2, 129.5, 127.9, 121.4, 113.3, 110.9, 55.3, 21.6, 14.2; HRMS calcd for $\mathrm{C}_{18} \mathrm{H}_{19} \mathrm{~N}_{2} \mathrm{O}_{3} \mathrm{~S}_{2}[\mathrm{M}+\mathrm{H}]^{+} 375.0832$, found 375.0831 .

3-甲硫基-5-对氟苯基-1-对甲苯磺酰基-1H-吡唑 (3d): 白色固体, m.p. 113.1 114.7 ${ }^{\circ} \mathrm{C} ;{ }^{1} \mathrm{H}$ NMR (400 $\left.\mathrm{MHz} \mathrm{CDCl}_{3}\right) \delta: 7.55(\mathrm{~d}, J=8.4 \mathrm{~Hz}, 2 \mathrm{H}), 7.42 \sim 7.34(\mathrm{~m}$, 2H), 7.22 (d, $J=8.0 \mathrm{~Hz}, 2 \mathrm{H}), 7.11(\mathrm{t}, J=8.4 \mathrm{~Hz}, 2 \mathrm{H}), 6.14$ $(\mathrm{s}, 1 \mathrm{H}), 2.55(\mathrm{~s}, 3 \mathrm{H}), 2.40(\mathrm{~s}, 3 \mathrm{H}) ;{ }^{13} \mathrm{C} \mathrm{NMR}(100 \mathrm{MHz}$, $\left.\mathrm{CDCl}_{3}\right) \delta: 163.52(\mathrm{~d}, J=249.8 \mathrm{~Hz}), 154.4,148.9,145.4$, 134.5, 131.7 (d, $J=8.5 \mathrm{~Hz}), 129.6,127.9,125.2$ (d, $J=3.5$ $\mathrm{Hz}), 115.0$ (d, $J=22.0 \mathrm{~Hz}), 111.3,21.7$, 14.2; ESI-HRMS calcd for $\mathrm{C}_{17} \mathrm{H}_{16} \mathrm{FN}_{2} \mathrm{O}_{2} \mathrm{~S}_{2}[\mathrm{M}+\mathrm{H}]^{+}$363.0632, found 363.0630 .

3-甲硫基-5-对氯苯基-1-对甲苯磺酰基- $1 H$-吡唑 (3e): 白色固体, m.p. 96.8 98.2 ${ }^{\circ} \mathrm{C} ;{ }^{1} \mathrm{H}$ NMR (400 MHz, $\left.\mathrm{CDCl}_{3}\right) \delta: 7.56(\mathrm{~d}, J=8.4 \mathrm{~Hz}, 2 \mathrm{H}), 7.40(\mathrm{~d}, J=8.4 \mathrm{~Hz}$, 2H), 7.34 (d, $J=8.4 \mathrm{~Hz}, 2 \mathrm{H}), 7.23$ (d, $J=8.0 \mathrm{~Hz}, 2 \mathrm{H}), 6.15$ (s, 1H), $2.55(\mathrm{~s}, 3 \mathrm{H}), 2.40(\mathrm{~s}, 3 \mathrm{H}) ;{ }^{13} \mathrm{C} \mathrm{NMR}(100 \mathrm{MHz}$, $\left.\mathrm{CDCl}_{3}\right) \delta: 154.6,148.8,145.4,135.8,134.4,131.1,129.6$,
128.2, 127.9, 127.6, 111.5, 21.7, 14.2; ESI-HRMS calcd for $\mathrm{C}_{17} \mathrm{H}_{16} \mathrm{ClN}_{2} \mathrm{O}_{2} \mathrm{~S}_{2}[\mathrm{M}+\mathrm{H}]^{+}$379.0336, found 379.0336.

3-甲硫基-5-对溴苯基-1-对甲苯磺酰基- $1 H$-吡唑 (3f): 白色固体, m.p. 103.6 105.1 ${ }^{\circ} \mathrm{C}$; ${ }^{1} \mathrm{H}$ NMR (400 $\left.\mathrm{MHz}, \mathrm{CDCl}_{3}\right) \delta: 7.57(\mathrm{~d}, J=2.4 \mathrm{~Hz}, 2 \mathrm{H}), 7.55(\mathrm{~d}, \quad J=$ $2.8 \mathrm{~Hz}, 2 \mathrm{H}), 7.27$ (d, $J=8.4 \mathrm{~Hz}, 2 \mathrm{H}), 7.23$ (d, $J=8.4 \mathrm{~Hz}$, $2 \mathrm{H}), 6.15(\mathrm{~s}, 1 \mathrm{H}), 2.54(\mathrm{~s}, 3 \mathrm{H}), 2.40(\mathrm{~s}, 3 \mathrm{H}) ;{ }^{13} \mathrm{C} \mathrm{NMR}$ $\left(100 \mathrm{MHz}, \mathrm{CDCl}_{3}\right) \delta: 154.6,148.8,145.4,134.5,131.3$, 131.1, 129.6, 128.1, 127.9, 124.1, 111.4, 21.7, 14.2; HRMS calcd for $\mathrm{C}_{17} \mathrm{H}_{16} \mathrm{BrN}_{2} \mathrm{O}_{2} \mathrm{~S}_{2}[\mathrm{M}+\mathrm{H}]^{+}$422.9831, found 422.9831 .

3-甲硫基-5-间甲基苯基-1-对甲苯磺酰基- $1 H$-吡唑 (3g): 白色固体, m.p. 79.2 80.9 ${ }^{\circ} \mathrm{C} ;{ }^{1} \mathrm{H}$ NMR (400 MHz, $\left.\mathrm{CDCl}_{3}\right) \delta: 7.57(\mathrm{~d}, J=8.4 \mathrm{~Hz}, 2 \mathrm{H}), 7.33 \sim 7.27(\mathrm{~m}, 2 \mathrm{H})$, $7.24 \sim 7.17(\mathrm{~m}, 4 \mathrm{H}), 6.13(\mathrm{~s}, 1 \mathrm{H}), 2.54(\mathrm{~s}, 3 \mathrm{H}), 2.40(\mathrm{~s}$, $6 \mathrm{H}) ;{ }^{13} \mathrm{C} \mathrm{NMR}\left(100 \mathrm{MHz}, \mathrm{CDCl}_{3}\right) \delta: 154.2,150.2,145.2$, $137.4,134.7,130.5,130.3,129.5,129.1,128.0,127.7$, 126.9, 111.1, 21.6, 21.3, 14.2; HRMS calcd for $\mathrm{C}_{18} \mathrm{H}_{19} \mathrm{~N}_{2} \mathrm{O}_{2} \mathrm{~S}_{2}[\mathrm{M}+\mathrm{H}]^{+}$359.0882, found 359.0881.

3-甲硫基-5-间氯苯基-1-对甲苯磺酰基- $1 H$-吡唑 (3h): 白色固体, m.p. 107.2 109.1 ${ }^{\circ} \mathrm{C} ;{ }^{1} \mathrm{H}$ NMR (400 $\left.\mathrm{MHz}, \mathrm{CDCl}_{3}\right) \delta: 7.61(\mathrm{~d}, J=8.4 \mathrm{~Hz}, 2 \mathrm{H}), 7.46(\mathrm{dt}, \quad J=$ 7.6, $1.6 \mathrm{~Hz}, 1 \mathrm{H}), 7.39(\mathrm{t}, J=8.0 \mathrm{~Hz}, 1 \mathrm{H}), 7.36 \sim 7.33(\mathrm{~m}$, 2H), 7.27 (d, $J=8.4 \mathrm{~Hz}, 2 \mathrm{H}), 6.19$ (s, 1H), 2.57 (s, 3H), $2.43(\mathrm{~s}, 3 \mathrm{H}) ;{ }^{13} \mathrm{C}$ NMR $\left(100 \mathrm{MHz}, \mathrm{CDCl}_{3}\right) \delta: 154.4,148.2$, $145.5,134.4,133.7,130.9,129.7,129.6,129.6,129.1$, 128.2, 127.9, 111.5, 21.7, 14.2; ESI-HRMS calcd for $\mathrm{C}_{17} \mathrm{H}_{16} \mathrm{ClN}_{2} \mathrm{O}_{2} \mathrm{~S}_{2}[\mathrm{M}+\mathrm{H}]^{+}$379.0336, found 379.0335.

3 -甲硫基-5-( $\beta$-萫基)-1-对甲苯磺酰基- $1 H$-吡唑(3i): 白色固体, m.p. 126.9 $128.4{ }^{\circ} \mathrm{C}$; ${ }^{1} \mathrm{H}$ NMR (400 MHz, $\left.\mathrm{CDCl}_{3}\right) \delta: 7.91 \sim 7.81(\mathrm{~m}, 4 \mathrm{H}), 7.58 \sim 7.52(\mathrm{~m}, 5 \mathrm{H}), 7.19$ $(\mathrm{d}, J=8.0 \mathrm{~Hz}, 2 \mathrm{H}), 6.24(\mathrm{~s}, 1 \mathrm{H}), 2.57(\mathrm{~s}, 3 \mathrm{H}), 2.38$ (s, 3H); ${ }^{13} \mathrm{C} \mathrm{NMR}\left(100 \mathrm{MHz}, \mathrm{CDCl}_{3}\right) \delta: 154.6,150.2,145.3,134.6$, $133.6,132.4,129.5,129.0,128.3,128.0,127.9,127.4$, 127.3, 127.1, 126.7, 126.6, 111.7, 21.7, 14.3; ESI-HRMS calcd for $\mathrm{C}_{21} \mathrm{H}_{19} \mathrm{~N}_{2} \mathrm{O}_{2} \mathrm{~S}_{2}[\mathrm{M}+\mathrm{H}]^{+}$395.0882, found 395.0882 .

3-甲硫基-5-(3,4-二氯苯基)-1-对甲苯磺酰基- $1 H$-吡 唑(3j): 白色固体, m.p. 124.8 126.2 ${ }^{\circ} \mathrm{C}$; ${ }^{1} \mathrm{H}$ NMR (400 $\left.\mathrm{MHz}, \mathrm{CDCl}_{3}\right) \delta: 7.61(\mathrm{~d}, J=8.0 \mathrm{~Hz}, 2 \mathrm{H}), 7.53$ (d, $J=8.4$ $\mathrm{Hz}, 1 \mathrm{H}), 7.46(\mathrm{~d}, J=2.0 \mathrm{~Hz}, 1 \mathrm{H}), 7.33 \sim 7.26(\mathrm{~m}, 3 \mathrm{H})$, $6.20(\mathrm{~s}, 1 \mathrm{H}), 2.57(\mathrm{~s}, 3 \mathrm{H}), 2.44(\mathrm{~s}, 3 \mathrm{H}) ;{ }^{13} \mathrm{C}$ NMR $(100$ $\left.\mathrm{MHz}, \mathrm{CDCl}_{3}\right) \delta: 154.6,147.2,145.7,134.3,134.0,132.2$, 131.3, 129.9, 129.7, 129.2, 129.1, 127.9, 111.6, 21.7, 14.2; ESI-HRMS calcd for $\mathrm{C}_{17} \mathrm{H}_{15} \mathrm{Cl}_{2} \mathrm{~N}_{2} \mathrm{O}_{2} \mathrm{~S}_{2} \quad[\mathrm{M}+\mathrm{H}]$ 
412.9947, found 412.9947.

3-甲硫基-5-对甲基苯基-1-苯磺酰基- $1 H$-吡唑(3k): 白色固体, m.p. 81.9 83.2 ${ }^{\circ} \mathrm{C} ;{ }^{1} \mathrm{H}$ NMR $(400 \mathrm{MHz}$, $\left.\mathrm{CDCl}_{3}\right) \delta: 7.71 \sim 7.66(\mathrm{~m}, 2 \mathrm{H}), 7.57(\mathrm{t}, J=7.6 \mathrm{~Hz}, 1 \mathrm{H})$, $7.45 \sim 7.40(\mathrm{~m}, 2 \mathrm{H}), 7.29(\mathrm{~d}, J=8.0 \mathrm{~Hz}, 2 \mathrm{H}), 7.23(\mathrm{~d}, J=$ $8.0 \mathrm{~Hz}, 2 \mathrm{H}), 6.13(\mathrm{~s}, 1 \mathrm{H}), 2.54(\mathrm{~s}, 3 \mathrm{H}), 2.43$ (s, 3H); ${ }^{13} \mathrm{C}$ NMR $\left(100 \mathrm{MHz}, \mathrm{CDCl}_{3}\right) \delta: 154.7,150.4,139.8,137.6$, 134.0, 129.7, 128.8, 128.6, 127.9, 126.2, 111.2, 21.5, 14.2; ESI-HRMS calcd for $\mathrm{C}_{17} \mathrm{H}_{17} \mathrm{~N}_{2} \mathrm{O}_{2} \mathrm{~S}_{2}[\mathrm{M}+\mathrm{H}]^{+} 345.0726$, found 345.0725

3-甲硫基-5-对甲基苯基-1-对甲氧基苯磺酰基- $1 H$ 吡坐(3I): 白色固体, m.p. 179.5 180.6 ${ }^{\circ} \mathrm{C} ;{ }^{1} \mathrm{H} \mathrm{NMR}$ $\left(400 \mathrm{MHz}, \mathrm{CDCl}_{3}\right) \delta: 7.62(\mathrm{~d}, J=9.2 \mathrm{~Hz}, 2 \mathrm{H}), 7.30$ (d, $J=$ $8.4 \mathrm{~Hz}, 2 \mathrm{H}), 7.22$ (d, $J=8.0 \mathrm{~Hz}, 2 \mathrm{H}), 6.87(\mathrm{~d}, J=9.2 \mathrm{~Hz}$, $2 \mathrm{H}), 6.12(\mathrm{~s}, 1 \mathrm{H}), 3.84(\mathrm{~s}, 3 \mathrm{H}), 2.54(\mathrm{~s}, 3 \mathrm{H}), 2.42$ (s, 3H); ${ }^{13} \mathrm{C} \mathrm{NMR}\left(100 \mathrm{MHz}, \mathrm{CDCl}_{3}\right) \delta: 164.0,154.2,150.2,139.6$, 130.3, 129.7, 129.0, 128.5, 126.4, 114.0, 111.0, 55.7, 21.4, 14.2; HRMS calcd for $\mathrm{C}_{18} \mathrm{H}_{19} \mathrm{~N}_{2} \mathrm{O}_{3} \mathrm{~S}_{2}[\mathrm{M}+\mathrm{H}]^{+} 375.0832$, found 375.0831 .

3-甲硫基-5-对甲基苯基-1-甲磺酰基- $1 H$-吡唑(3m): 白色固体, m.p. 89.9 91.1 ${ }^{\circ} \mathrm{C} ;{ }^{1} \mathrm{H}$ NMR (400 MHz, $\left.\mathrm{CDCl}_{3}\right) \delta: 7.36(\mathrm{~d}, J=8.0 \mathrm{~Hz}, 2 \mathrm{H}), 7.22(\mathrm{~d}, J=7.6 \mathrm{~Hz}$, 2H), 6.25 (s, 1H), 3.18 (s, 3H), 2.60 (s, 3H), 2.39 (s, 3H); ${ }^{13} \mathrm{C} \mathrm{NMR}\left(100 \mathrm{MHz}, \mathrm{CDCl}_{3}\right) \delta: 153.9,149.7,139.9,129.5$, 128.7, 125.8, 110.3, 41.7, 21.4, 14.2; ESI-HRMS calcd for $\mathrm{C}_{12} \mathrm{H}_{15} \mathrm{~N}_{2} \mathrm{O}_{2} \mathrm{~S}_{2}[\mathrm{M}+\mathrm{H}]^{+}$283.0569, found 283.0569.

3-甲硫基-5-对甲基苯基-1-乙磺酰基- $1 H$-吡唑(3n): 白色固体, m.p. 132.5 134.3 ${ }^{\circ} \mathrm{C}$; ${ }^{1} \mathrm{H}$ NMR (400 MHz, $\left.\mathrm{CDCl}_{3}\right) \delta: 7.35(\mathrm{~d}, J=8.0 \mathrm{~Hz}, 2 \mathrm{H}), 7.21(\mathrm{~d}, J=8.0 \mathrm{~Hz}$, 2H), 6.23 (s, 1H), 3.38 (q, J=7.2 Hz, 2H), 2.60 (s, 3H), 2.39 (s, 3H), 1.18 (t, J=7.2 Hz, 3H); ${ }^{13} \mathrm{C}$ NMR (100 MHz, $\left.\mathrm{CDCl}_{3}\right) \delta: 153.8,150.5,139.7,129.6,128.6,125.9,109.9$, 49.2, 21.4, 14.3, 7.5; HRMS calcd for $\mathrm{C}_{13} \mathrm{H}_{17} \mathrm{~N}_{2} \mathrm{O}_{2} \mathrm{~S}_{2}[\mathrm{M}+$ $\mathrm{H}]^{+}$297.0726, found 297.0725.

3-甲硫基-5-对甲基苯基-1-茮基磺酰基- $1 H$-吡唑 (3o): 白色固体, m.p. 184.3 185.8 ${ }^{\circ} \mathrm{C} ;{ }^{1} \mathrm{H}$ NMR (400 $\left.\mathrm{MHz}, \mathrm{CDCl}_{3}\right) \delta: 7.37(\mathrm{t}, J=7.6 \mathrm{~Hz}, 1 \mathrm{H}), 7.31 \sim 7.24(\mathrm{~m}$, $2 \mathrm{H}), 7.01(\mathrm{~d}, J=8.0 \mathrm{~Hz}, 4 \mathrm{H}), 6.71(\mathrm{~d}, J=8.0 \mathrm{~Hz}, 2 \mathrm{H}), 6.01$ $(\mathrm{s}, 1 \mathrm{H}), 4.59$ (s, 3H), $2.63(\mathrm{~s}, 3 \mathrm{H}), 2.32(\mathrm{~s}, 2 \mathrm{H}) ;{ }^{13} \mathrm{C} \mathrm{NMR}$ $\left(100 \mathrm{MHz}, \mathrm{CDCl}_{3}\right) \delta: 154.0,151.4,139.3,130.8,129.3$, 129.2, 128.8, 128.1, 126.7, 125.6, 109.6, 60.2, 21.4, 14.3; ESI-HRMS calcd for $\mathrm{C}_{18} \mathrm{H}_{19} \mathrm{~N}_{2} \mathrm{O}_{2} \mathrm{~S}_{2}[\mathrm{M}+\mathrm{H}]^{+} 359.0882$, found 359.0883 .

3-乙硫基-5-苯基-1-对甲苯磺酰基- $1 H$-吡唑(3p). 白 色固体, m.p. $122.0 \sim 123.9{ }^{\circ} \mathrm{C}$; ${ }^{1} \mathrm{H}$ NMR (400 MHz,
$\left.\mathrm{CDCl}_{3}\right) \delta: 7.98(\mathrm{~d}, J=8.4 \mathrm{~Hz}, 2 \mathrm{H}), 7.79(\mathrm{dd}, J=8.0,1.6$ $\mathrm{Hz}, 2 \mathrm{H}), 7.42 \sim 7.34(\mathrm{~m}, 3 \mathrm{H}), 7.30(\mathrm{~d}, J=8.0 \mathrm{~Hz}, 2 \mathrm{H})$, 6.43 (s, 1H), 2.99 (q, J=7.2 Hz, 2H), 2.39 (s, 3H), 1.38 (t, $J=7.2 \mathrm{~Hz}, 3 \mathrm{H}) ;{ }^{13} \mathrm{C} \mathrm{NMR}\left(100 \mathrm{MHz}, \mathrm{CDCl}_{3}\right) \delta: 155.4$, $145.5,143.5,134.6,131.3,129.8,129.2,128.6,128.2$, 126.3, 106.5, 28.8, 21.7, 13.5; HRMS calcd for $\mathrm{C}_{18} \mathrm{H}_{19} \mathrm{~N}_{2} \mathrm{O}_{2} \mathrm{~S}_{2}[\mathrm{M}+\mathrm{H}]^{+}$359.0882, found 359.0882.

3-乙硫基-5-对氯苯基-1-对甲苯磺酰基- $1 H$-吡唑 (3q): 白色固体, m.p. 139.8 141.7 ${ }^{\circ} \mathrm{C}$; ${ }^{1} \mathrm{H}$ NMR (400 $\left.\mathrm{MHz} \mathrm{CDCl}_{3}\right) \delta: 7.55(\mathrm{~d}, J=8.4 \mathrm{~Hz}, 2 \mathrm{H}), 7.42 \sim 7.32(\mathrm{~m}$, $4 \mathrm{H}), 7.22$ (d, $J=8.0 \mathrm{~Hz}, 2 \mathrm{H}), 6.15$ (s, 1H), 3.08 (q, $J=7.2$ $\mathrm{Hz}, 2 \mathrm{H}), 2.39$ (s, 3H), 1.32 (t, $J=7.2 \mathrm{~Hz}, 3 \mathrm{H}) ;{ }^{13} \mathrm{C} \mathrm{NMR}$ $\left(100 \mathrm{MHz}, \mathrm{CDCl}_{3}\right) \delta: 153.6,148.6,145.5,135.8,134.5$, 131.1, 129.6, 128.1, 127.9, 127.7, 112.2, 25.9, 21.7, 14.6; HRMS calcd for $\mathrm{C}_{18} \mathrm{H}_{18} \mathrm{ClN}_{2} \mathrm{O}_{2} \mathrm{~S}_{2}[\mathrm{M}+\mathrm{H}]^{+}$393.0493, found 393.0492 .

3-甲硫基-5-甲基-4-乙酰基-1-对甲苯磺酰基- $1 H$-吡 唑(3r): 白色固体, m.p. 106.8 108.0 ${ }^{\circ} \mathrm{C} ;{ }^{1} \mathrm{H}$ NMR (400 $\left.\mathrm{MHz}, \mathrm{CDCl}_{3}\right) \delta: 7.88(\mathrm{~d}, J=8.4 \mathrm{~Hz}, 2 \mathrm{H}), 7.34$ (d, $J=8.0$ $\mathrm{Hz}, 2 \mathrm{H}), 2.81$ (s, 3H), 2.45 (s, 3H), 2.43 (s, 3H), 2.42 (s, $3 \mathrm{H}) ;{ }^{13} \mathrm{C}$ NMR $\left(100 \mathrm{MHz}, \mathrm{CDCl}_{3}\right) \delta: 186.8,151.6,146.1$, 145.9, 134.61, 130.1, 128.0, 121.8, 21.7, 15.0, 12.6, 11.7; HRMS calcd for $\mathrm{C}_{14} \mathrm{H}_{17} \mathrm{~N}_{2} \mathrm{O}_{3} \mathrm{~S}_{2}[\mathrm{M}+\mathrm{H}]^{+} 325.0675$, found 325.0676 .

辅助材料(Supporting Information) 所有产物的 ${ }^{1} \mathrm{H}$ $\mathrm{NMR}$ 和 ${ }^{13} \mathrm{C} \mathrm{NMR}$ 图谱及化合物 3a 的单晶测试. 这些材 料可以免费从本刊网站(http://sioc-journal.cn/)上下载.

\section{References}

[1] (a) Gulia, N.; Daugulis, O. Angew. Chem., Int. Ed. 2017, 56, 3630. (b) Lee, W.-C. C.; Shen, Y.; Gutierrez, D. A.; Li, J. J. Org. Lett. 2016, $18,2660$.

(c) Boerth, J. A.; Hummel, J. R.; Ellman, J. A. Angew. Chem., Int. Ed. 2016, 55, 12650.

[2] (a) Olguin, J.; Brooker, S. Coord. Chem. Rev. 2011, 255, 203. (b) Bailey, W. D.; Luconi, L.; Tossin, A.; Yakhvarov, D.; Flowers, S. E.; Kaminsky, W.; Kemp, R. A.; Giambastiani, G.; Goldberg, K. I. Organometallics 2015, 34, 3998.

(c) Kuwata, S.; Ikariya, T. Chem. Commun. 2014, 50, 14290.

(d) Sinha, A. K.; Vigalok, A.; Rawat, V. Tetrahedron Lett. 2019, 60, 796.

[3] (a) Yin, P.; He, C.; Shreeve, J. M. J. Mater. Chem. A 2016, 4, 1514. (b) Dalinger, I. L.; Kormanov, A. V.; Suponitsky, K. Y.; Muravyev, N. V.; Sheremetev, A. B. Chem.-Asian J. 2018, 13, 1165.

(c) Taydakov, I. V.; Akkuzina, A. A.; Avetisov, R. I.; Khomyakov, A. V.; Saifutyarov, R. R.; Avetissov, I. C. J. Lumin. 2016, 177, 31.

(d) Li, Y.; Chang, P.; Hu, J.; Chen, T.; Wang, B.; Wang, Y.; Wang, B. Curr. Org. Chem. 2018, 24, 1407.

[4] (a) Yang, W.; Li, Y.; Ai, Y.; Obianom, O. N.; Guo, D.; Yang, H.; Sakamuru, S.; Xia, M.; Shu, Y.; Xue, F. J. Med. Chem. 2019, 62, 11151

(b) Ahmed, W.; Yan, X.; Hu, D.; Adnan, M.; Tang, R.-Y.; Cui, Z.-N. 
Bioorg. Med. Chem. 2019, 27, No. UNSP 115048.

(c) Geng, R.; Zhao, Y.; Li, Y.; Liu, X.; Wang, M. Chin. J. Org. Chem. 2019, 39, 3574 (in Chinese).

(耿瑞, 赵宇, 李益豪, 刘釒否, 王明安, 有机化学, 2019, 39, 3574.)

(d) Monteiro, M. E.; Lechuga, G.; Lara, L. S.; Souto, B. A.; Vigano, M. G.; Bourguignon, S. C.; Calvet, C. M.; Oliveira, F. O. R.; Alves, C. R.; Souza-Silva, F.; Santos, M. S.; Pereira, M. C. S. Eur. J. Med. Chem. 2019, 182, 111610

(e) Fu. Q.; Cai, P.-P.; Cheng, L.; Zhong, L.-K.; Tan, C.-X.; Shen, Z.-H.; Han, L.; Xu, T.-M.; Liu, X.-H. Pest Manage. Sci. 2019, 76, 868.

(f) Zhong, L.; Jiang, T.; Zhang, F.; Fu, Q.; Liu, X.; Xu, T.; Ding, C.; Chen, J.; Yuan, J.; Tan, C. Chin. J. Org. Chem. 2019, 39, 2655 (in Chinese).

(钟良坤, 江涛, 张凡, 付庆, 刘幸海, 许天明, 丁成荣, 陈杰, 袁静, 谭成侠, 有机化学, 2019, 39, 2655.)

(g) Hassan, G. S.; Rahman, D. E. A.; Abdelmajeed, E. A.; Refaey, R. H.; Salem, M. A.; Nissan, Y. M. Eur. J. Med. Chem. 2019, 171, 332.

(h) Li, W.; Li, J.; Shen, H.; Cheng, J.; Li, Z.; Xu, X. Chin. Chem. Lett. 2018, 29, 911.

(i) Bondock, S.; Fadaly, W.; Metwally, M. A. Eur. J. Med. Chem. 2010, 45, 3692.

(j) Wang, Y.; Xu, W.; Shao, S.; Xie, Y.; Wang, J. Chin. J. Chem. 2011, 29, 2039 .

[5] (a) Fustero, S.; Sánchez-Roselló, M.; Barrio, P.; Simón-Fuentes, A. Chem. Rev. 2011, 111, 6984

(b) Janin, Y. L. Chem. Rev. 2012, 112, 3924.

(c) Maddila, S.; Jonnalagadda, S. B.; Gangu, K. K.; Maddila, S. N. Curr. Org. Chem. 2017, 14, 634.

[6] (a) Heller, S. T.; Natarajan, S. R. Org. Lett. 2006, 8, 2675.

(b) Jansa, J.; Schmidt, R.; Mamuye, A. D.; Castoldi, L.; Roller, A.; Pace, V.; Holzer, W. Beilstein J. Org. Chem. 2017, 13, 895.

(c) Zhang, J.; Shao, Y.; Wang, H.; Luo, Q.; Chen, J.; Xu, D.; Wan, X. Org. Lett. 2014, 16, 3312.

[7] (a) Guo, Y.; Wang, D.; Wei, L.; Wan, J.-P. J. Org. Chem. 2019, 84, 2984.

(b) Tian, L.; Wan, J.-P.; Sheng, S. Chem CatChem 2020, 12, 2533

(c) Zhang, Q.; Hu, B.; Zhao, Y.; Zhao, S.; Wang, Y.; Zhang, B.; Yan, S.; Yu, F. Eur. J. Org. Chem. 2020, 1154.

[8] Pearce, A. J.; Harkins, R. P.; Reiner, B. P.; Wotal, A. C.; Dunscomb, R. J.; Tonks, I. A. J. Am. Chem. Soc. 2020, 142, 4390.

[9] (a) Fricero, P.; Bialy, L.; Brown, A. W.; Czechtizky, W.; Méndez, M.; Harrity, J. P. A. J. Org. Chem. 2017, 82, 1688.

(b) Chen, Y.; Zhu, J.; Zhao, S. Chin. J. Org. Chem. 2019, 39, 1923 (in Chinese).

(陈樱，祝家楠，赵圣印，有机化学, 2019, 39, 1923.)

(c) Gilfillan, L.; Artschwager, R.; Harkiss, A. H.; Liskamp, R. M. J.; Sutherland, A. Org. Biomol. Chem. 2015, 13, 4514.

[10] (a) Das, P.; Gondo, S.; Tokunaga, E.; Sumii, Y.; Shibata, N. Org. Lett. 2018, 20, 558.

(b) Shao, Y.; Zheng, H.; Qian, J.; Wan, X. Org. Lett. 2018, 20, 2412 .

(c) Zeng, J.-L.; Chen, Z.; Zhang, F.-G.; Ma, J.-A. Org. Lett. 2018, 20,4562 .

[11] (a) Shu, W.-M.; Zheng, K.-L.; Ma, J.-R.; Sun, H.-Y.; Wang, M.; Wu, A.-X. Org. Lett. 2015, 17, 1914.

(b) Abu, T. K.; Arindam, G.; Sidick, B. R.; Mohammad, H. M. Asian J. Org. Chem. 2013, 2, 126.

[12] (a) Neumann, J. J.; Suri, M.; Glorius, F. Angew. Chem., Int. Ed.
2010, 49, 7790.

(b) Li, X.; He, L.; Chen, H.; Wu, W.; Jiang, H. J. Org. Chem. 2013, $78,3636$.

(c) Zhang, T.; Bao, W. J. Org. Chem. 2013, 78, 1317.

[13] (a) Pérez-Aguilar, M. C.; Valdés, C. Angew. Chem., Int. Ed. 2013, 52,7219 .

(b) Deng, X.; Mani, N. S. Org. Lett. 2008, 10, 1307.

(c) Kong, Y.; Tang, M.; Wang, Y. Org. Lett. 2014, 16, 576.

(d) Fang, Z.; Liu, J.; Qiao, Y. Chin. J. Org. Chem. 2018, 38, 1985 (in Chinese).

(房智兴, 刘巨艳, 乔艳红, 有机化学, 2018, 38, 1985.)

[14] (a) Fan, Z.-P.; Li, X.-Y.; Luo, X.-E.; Fei, X.; Sun, B.; Chen, L.-C.; Shi, Z.-F.; Sun, C.-L.; Shao, X.; Zhang, H. Adv. Funct. Mater. 2017, 27, 1702318

(b) Zhang, D.-W.; Yang, Y.; Yao, F.; Yu, Q.-Y.; Dai, S.-J. J. Nat. Med. 2012, 66, 362 .

(c) Appetcchi, G. B.; D’Annibale, A.; Santilli, C.; Genova, E.; Lombardo, L.; Navarra, M. A.; Panero, S. Electrochem. Commun. 2016, 63, 26

(d) Yao, J.; Chen, J.; He, Z.; Sun, W.; Fang, H.; Xu, W. Med. Chem. Res. 2013, 22, 3959.

[15] (a) Deng, L.; Cao, X.; Liu, Y.; Wan, J.-P. J. Org. Chem. 2019, 84, 14179 .

(b) Gao, Y.; Liu, Y.; Wan, J.-P. J. Org. Chem. 2019, 84, 2243.

(c) Zhong, S.; Liu, Y.; Cao, X.; Wan, J.-P. Chem CatChem 2017, 9, 465.

(d) Li, Y.; Wan, J.-P.; Wen, C. Tetrahedron 2017, 73, 2323.

(e) Guo, Y.; Xiang, Y.; Wei, L.; Wan, J.-P. Org. Lett. 2018, 20, 3971.

(f) Peng, S.; Song, Y.; He, J.-Y.; Tang, S.-S.; Tan, J.-X.; Cao, Z.; Lin, Y.-W.; He, W.-M. Chin. Chem. Lett. 2019, 30, 2287.

(g) Xie, L.-Y.; Fang, T.-G.; Tan, J.-X.; Zhang, B.; Cao, Z.; Yang, L.-H.; He, W.-M. Green Chem. 2019, 21, 3858.

(h) Xu, X.-M.; Chen, D.-M.; Wang, Z.-L. Chin. Chem. Lett. 2020, 31,49 .

(i) Dong, D.; Chen, W.; Chen, D.; Li, L.; Li, G.; Wang, Z.; Deng, Q.; Long, S. Chin. J. Org. Chem. 2019, 39, 3190 (in Chinese).

(董道青, 陈文静, 陈德茂, 李丽霞, 李光辉, 王祖利, 邓企, 龙 姝, 有机化学, 2019, 39, 3190.)

(j) Wang, Y.; Liu, Y. Acta Chim. Sinica 2019, 77, 418 (in Chinese). (王昱望，刘云云，化学学报, 2019, 77, 418.)

(k) Liu, Y.; Xiong, J.; Wei, L. Chin. J. Org. Chem. 2017, 37, 1667 (in Chinese)

(刘云云, 熊进, 韦丽, 有机化学, 2017, 37, 1667.)

(1) Li, L.-X.; Dong, D.-Q.; Hao, S.-H.; Wang, Z.-L. Tetrahedron Lett. 2018, 59, 1517.

[16] (a) Huang, L.; Wu, J.; Hu, J.; Bi, Y.; Huang, D. Tetrahedron Lett. 2020, 61, 151363 .

(b) Wang, M.; Shi, L.; Li, Y.; Liu, Q.; Pan, L. J. Org. Chem. 2019, $84,9603$.

(c) Deng, L.; Liu, Y. ACS Omega 2018, 3, 11890.

(d) Sorabad, G. S.; Maddani, M. R. New J. Chem. 2019, 43, 5996.

(e) Garg, P.; Singh, A. Org. Lett. 2018, 20, 1320.

(e) Fang, Z.; Liu, J.; Liu, Q.; Bi, X. Angew. Chem., Int. Ed. 2014, 53, 7209 .

[17] Peruncheralathan, S.; Khan, T. A.; Ila, H.; Junjappa, H. J. Org. Chem. 2005, 70, 10030.

[18] He, J.; Man, Z.; Shi, Y.; Li, C.-Y. J. Org. Chem. 2015, 80, 4816.

[19] (a) Wan, J.-P.; Hu, D.; Bai, F.; Wei, L.; Liu, Y. RSC Adv. 2016, 6, 73132.

(b) Backes, G. L.; Jursic, B. S.; Neumann, D. M. Bioorg. Med. Chem. 2015, 23, 3397. 